\title{
Low-Temperature MBE Grown GaAs for Pulsed THz Radiation Applications
}

\author{
R. Adomavičius, S. Balakauskas, K. Bertulis, A. Geižutis, \\ G. Molis and A. Krotkus* \\ Semiconductor Physics Institute, A. Goštauto 11, 01108 Vilnius, Lithuania
}

\begin{abstract}
Attempts to optimize recombination characteristics of low-temperature MBE grown GaAs layers for their use in terahertz radiation devices are described and the characteristics of this material are compared with its alternative - As-ion implanted GaAs crystals.

PACS numbers: 42.65.Re, 07.57.Hm, 78.47.+p
\end{abstract}

\section{Introduction}

The coherent generation and detection of terahertz $(\mathrm{THz})$ radiation using ultrashort laser pulses and photoconductive antennae have been intensively studied during the last decade. The best results were achieved when low-temperature MBE grown GaAs (LTG GaAs) or ion-implanted GaAs layers were used as a basis for $\mathrm{THz}$ emitters and detectors. The ultimate performance of these devices depends on carrier mobilities and their trapping times in semiconductor layers; therefore the determination of those parameters is of a critical importance. In the present work, we will describe the characterization of GaAs layers with ultrafast carrier recombination and the results obtained on $\mathrm{THz}$ radiation components manufactured from these layers.

\section{Experimental techniques}

Epitaxial LTG GaAs layers were grown on semi-insulating GaAs substrates in a solid-state molecular beam epitaxy (MBE) system. During the growth, $\mathrm{As}_{4} / \mathrm{Ga}$ beam-equivalent pressure ratio was kept equal to 10 and the substrate

*corresponding author; e-mail: krotkus@pfi.lt 
temperature was varied in the range of $240-270^{\circ} \mathrm{C}$. The layers underwent an after-growth anneal in the rapid thermal annealing chamber; the anneal temperatures were from 450 to $900^{\circ} \mathrm{C}$ and the anneal time was $30 \mathrm{~s}$. Moreover, As-ion implanted (the ion energy was $2 \mathrm{MeV}$, the ion dose was $10^{16} \mathrm{~cm}^{-2}$ ), $2 \mu \mathrm{m}$ thick epitaxially lifted-off layers of GaAs were investigated for comparison. It is known [1] that high energy ion implantation in GaAs results in nonstoichiometric disturbance and leads to material characteristics similar to LTG GaAs.

Electron dynamics was characterized by employing Ti:sapphire laser pulses (150 fs, $76 \mathrm{MHz}, 815 \mathrm{~nm}$ ). Optical pump — THz probe technique has been used for the electron trapping time measurement and the electron mobility estimation. $\mathrm{THz}$ transients were generated and detected by a pair of photoconductive antennae from LTG GaAs; both the pump and the probe signals were overlapping at $300 \mu \mathrm{m}$ diameter aperture that was placed in contact with the samples surface. The basic part of this system was the high-power THz emitter from LTG GaAs with a large signal-to-noise ratio. The reduction of the $\mathrm{THz}$ power transmitted through the sample under investigation due to its absorption by optically excited electrons was easily measurable even at their densities as low as $10^{16} \mathrm{~cm}^{-3}$.

For the hole trapping time measurement, a novel optical probe - mid-infrared (MIR) (the wavelength of $9 \mu \mathrm{m}$ ) probe technique [2] was used. The latter wavelength corresponds to the resonance transitions between the heavy and light hole valence bands, therefore, the light absorption by the free holes at the probe wavelength is at least 20 times larger than the free-electron absorption.

\section{Comparison of LTG and ion-implanted GaAs}

It has been demonstrated before [1] that the electron trapping characteristics in GaAs crystals implanted with high energy As and other heavy ions are similar to those of LTG GaAs. Electron trapping times in ion-implanted material could be easily reduced to 1 ps and shorter values; this material parameter becomes larger after thermal anneal at temperatures higher than $550^{\circ} \mathrm{C}$. In the case of the hole trapping times no such comparison was available, yet. Figure 1 shows the photoexcited hole dynamics measured by the optical pump - MIR probe technique on LTG GaAs and arsenic ion-implanted GaAs samples. Both samples were annealed at $600^{\circ} \mathrm{C}$ and had electron trapping times of a similar magnitude ( 0.5 and $1 \mathrm{ps,} \mathrm{respectively);} \mathrm{the} \mathrm{hole} \mathrm{trapping} \mathrm{rates} \mathrm{in} \mathrm{those} \mathrm{samples} \mathrm{were} \mathrm{also}$ similar to each other. This fact is an additional confirmation of the similarity of the physical mechanisms leading to the ultrafast carrier recombination in LTG and As-ion implanted GaAs.

Neutral and ionized As-antisite donor defects were proposed as the hole and, respectively, the electron traps in LTG GaAs [3]. The relative ionization level of these defects depends on their charge compensation by acceptors. In the case of LTG GaAs, the main acceptors are gallium vacancies. The latter defects are 


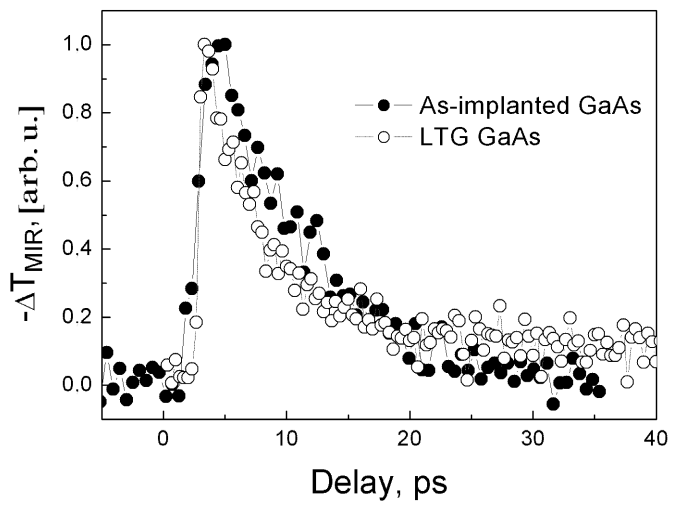

Fig. 1. Optical pump induced change of the MIR transmission for two nonstoichiometric GaAs samples. Hole trapping time determined from these experimental traces is equal to $\sim 7$ ps.

also the only possible acceptors that can be introduced in GaAs by the As-ion implantation.

One has to point out that nonstoichiometry related defects created by the heavy ion implantation are distributed non-homogeneously even at the distances smaller than the ion stop range [4]. This inhomogeneity leads to a different carrier trapping dynamics in different regions of the sample, which is evidenced by experimental traces presented in Fig. 2. The absorption length of the optical pump pulse was $\sim 0.8 \mathrm{~mm}$, therefore, in these traces $\mathrm{THz}$ probe pulses effectively sampled the electron density decay in two halves of the $2 \mu \mathrm{m}$ thick GaAs sample. As it can be seen from this figure, the characteristic time of this decay is close to the temporal resolution of the experiment ( $0.7 \mathrm{ps})$ at one side of the sample and much longer $(\sim 20 \mathrm{ps})$ at the opposite side.

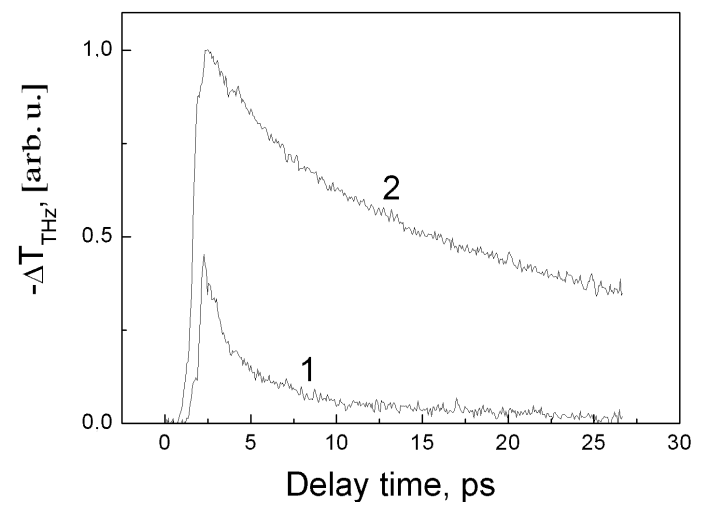

Fig. 2. Optical pump induced change of $\mathrm{THz}$ transmission for $2 \mathrm{MeV}$ As-implanted GaAs sample. Pump laser pulses were illuminating the implanted surface (1) and the back-surface (2) of the lifted-off from the substrate, $2 \mu \mathrm{m}$ thick GaAs layer. 


\section{4. $\mathrm{THz}$ radiation devices from LTG GaAs}

Photoconductive antennae for $\mathrm{THz}$ radiation emitters and detectors were manufactured from LTG GaAs grown and annealed at various temperatures. The photosensitive gap between the metal electrodes was $80 \mu \mathrm{m}$ for the emitters and $5 \mu \mathrm{m}$ for the detectors. Electrode structures were deposited either directly on the LTG GaAs layers or had an intermediate heavily p-doped GaAs contact layer.

The largest bandwidth of the detectors $4 \mathrm{THz}$ (when activated by $150 \mathrm{fs}$ duration laser pulses) was achieved when a LTG GaAs layer grown at $240^{\circ} \mathrm{C}$ temperature was used, whereas the largest magnitude of the emitted $\mathrm{THz}$ signals was obtained with the emitter made from the layer annealed at lower than usual $\left(\sim 450^{\circ} \mathrm{C}\right)$ temperature. In this case, average field in the emitter with metalsemiconductor-metal contact structure was close to its limit imposed by the effect of impact ionization in $\mathrm{GaAs}(>250 \mathrm{kV} / \mathrm{cm})$. Average $\mathrm{THz}$ power for this device was large enough $(\sim 5 \mu \mathrm{W})$ to be measured by a pyroelectric detector at room temperature.

\section{Acknowledgment}

This research was supported by NATO Science Committee (Projects SfP-977978 and SfP-974476 ).

\section{References}

[1] A. Krotkus, S. Marcinkevičius, J. Jasinski, M. Kaminska, H.H. Tan, C. Jagadish, Appl. Phys. Lett. 66, 3304 (1995).

[2] R. Adomavičius, A. Krotkus, K. Bertulis, V. Sirutkaitis, R. Butkus, A. Piskarskas, Appl. Phys. Lett. 83, 5304 (2003).

[3] A. Krotkus, K. Bertulis, M. Kaminska, K. Korona, A. Wolos, J. Siegert, S. Marcinkevičius, J.-F. Roux, J.-L. Coutaz, IEE Proc. J. Optoelectron. 149, 111 (2002).

[4] Y. Zhang, E. Ding, T. Zhang, Nucl. Instrum. Methods Phys. Res. B 152, 307 (1999). 